\title{
Human Embryonic Behavior Observed with Time-Lapse Cinematography
}

Yasuyuki Mio ${ }^{1 *}$, Kyoko Iwata ${ }^{1}$, Keitaro Yumoto ${ }^{1}$ and Kazuo Maeda ${ }^{2}$

${ }^{1}$ Reproductive Centre, Mio Fertility Clinic, 2-1-1 Kuzumo-Minami, Yonago, Japan

${ }^{2}$ Faculty of Medicine, Tottori University, Japan

\begin{abstract}
To clarify developmental behavior in human embryos, we observed oocytes with in vitro fertilization and intracytoplasmic sperm injection and donated embryos up to 5 days using our original time-lapse cinematography (TLC) system. The TLC analyses demonstrated several novel aspects and events that were not visible by intermittent stillframe microscopy. Here, we present the entire fertilization process in which we founded the fertilization cone, cytoplasmic flare, dynamic change of nuclear precursor body and cytoplasmic halo. We also demonstrated the occurrence of zygotes with a single pronucleus (PN) and uneven 2PN, fragmentation, blastocoel collapse during the blastocyst stage, and the hatching pattern of expanded blastocysts. Observing such morphokineses in human embryos will enhance our understanding of human embryonic behavior up to preimplantation.
\end{abstract}

Keywords: Time-lapse cinematography; Human embryonic development; Fertilization process; Hatching; Fragmentation; A single PN; Uneven 2PN; Blasotocoel collapse

\section{Introduction}

Assisted reproductive technologies (ART) have enabled the in vitro observation of a human embryo as it develops; however, evaluating the morphokinetics of embryonic development in still images remains difficult. In 1997, Payne et al. [1] developed time-lapse video cinematography (TLC) to overcome the limitations of intermittent observation by providing high-resolution, continuous imaging, in which various cellular events could be distinguished in real time and monitored during the observation period. However, this system was restricted to an observation period of 17-20 hours. Based on Payne's work, we developed a new TLC system that maintains optimal and stable culture conditions on the microscope stage for periods of up to a week [2,3]. Using this system, we have observed human embryonic development from the fertilization process to the hatched blastocyst stage. Herein, we present several novel aspects and events identified using our system during that developmental period.

\section{Materials and Methods}

We developed a new system for TLC, based on the concept of Payne et al. [1-3], wherein the optimal embryo culture conditions (temperature: $37 \pm 0.3^{\circ} \mathrm{C}, \mathrm{pH}: 7.45 \pm 0.03$ ) are maintained by continuously monitoring and adjusting the flow of $\mathrm{CO}_{2}(40 \mathrm{~mL} /$ $\mathrm{min})$, the air temperature in the large chamber $\left(38.0^{\circ} \mathrm{C}\right)$, and the thermoplate temperature on the microscope stage $\left(41.8^{\circ} \mathrm{C}\right)$. The inverted microscope is equipped with a CCD digital camera (Roper Scientific Photometrics, Tuscon, AZ) connected to the computer and display by Meta Morph (Universal Imaging Co, Downingtown, PA). In this study, digital images of the cultured embryos were acquired for 2-5 days using an exposure time of $50 \mathrm{~ms}$. In total, 2000-8000 images were taken during the observation period. The time interval of image storage was $10 \mathrm{sec}$ during fertilization and $2 \mathrm{~min}$ thereafter until the end of the observation.

In this study, we used our TLC system to observe 1) oocytes for clinical use and 2) embryos for research work. In oocytes for clinical use, after receiving informed consent from the couples, one oocyte out of 10 or more oocytes retrieved in each patient was randomly selected for TLC observation.

In oocytes derived by in vitro fertilization (IVF), the cumulus cells were mechanically removed so as not to damage the tail of the sperm that had penetrated the zona pellucida (ZP) at one hour after insemination with 50,000 of the husband's motile sperm. The oocyte was transferred into a microdrop of culture medium $(5 \mu$, Fertilization medium $^{\circledR}$, COOK, Australia) covered by mineral oil (SAGE, USA), and the site of sperm penetration into the ZP was carefully identified by micromanipulation (Narishige, Japan). The microscope was focussed on the sperm within the ZP that was penetrated most deeply and TLC observations were carried out at 10 -second intervals until the leading sperm penetrated $\mathrm{ZP}$ and attached to the oocyte membrane. Thereafter, TLC was continued for approximately 40 hours at 2 -minute intervals. Oocytes for intracytoplasmic sperm injection (ICSI) were transferred immediately into a microdrop of culture medium covered by mineral oil and TLC observation was commenced for up to 40 hours at 2-minute time intervals. At the end of the observation period, goodquality embryos developed in both CIVF and ICSI were cryopreserved for future clinical use.

On the other hand, the donated frozen/thawed day-2, -3 embryos for research work were thawed and cultured for 3 to 5 hours to assess viability and embryo quality. After the assessment of embryos, the earlystage, good-quality embryos were transferred into $5 \mu \mathrm{l}$ of the culture medium covered by mineral oil and TLC observation was commenced for up to 5 days at 2- to 5- minute time intervals. These TLC observations were approved by the ethical committee of JISART (Japanese Institution for Standardizing Assisted Reproductive Technology).

\section{Results}

\section{Fertilization process (representative oocyte) in IVF (Supplementary Movie 1)}

We previously visualized the entire process of fertilization of a

*Corresponding author: Yasuyuki Mio, Reproductive Centre, Mio Fertility Clinic 2-1-1 Kuzumo-Minami, Yonago, Japan, Tel: 81-859-22-6856; E-mail: yasmio@mfc.or.jp

Received December 16, 2013; Accepted January 04, 2014; Published January 10,2014

Citation: Mio Y, Iwata K, Yumoto K, Maeda K (2014) Human Embryonic Behavior Observed with Time-Lapse Cinematography. J Health Med Informat 5: 143. doi:10.4172/2157-7420.1000143

Copyright: (c) 2014 Mio Y, et al. This is an open-access article distributed under the terms of the Creative Commons Attribution License, which permits unrestricted use, distribution, and reproduction in any medium, provided the original author and source are credited. 
human oocyte following IVF and up to second cleavage by TLC [2,3]. This initial study also revealed several novel phenomena occurring during fertilization and cleavage [4]. In this study we further analyzed these novel phenomena by TLC.

Fertilization cone (FC) (Supplementary Movie 2): In other species including echinoderms $[5,6]$ and rodents $[7,8]$, the FC first appears during the fertilization process wherein it plays an important role. In the sea urchin oocytes, the FC was closely associated with the polyspermic block through depolarization of the oocyte membrane [5,6], while in rodent oocytes, the FC was detectable from 2 to 3 hours postinsemination prior to the decondensation of sperm chromatin, and it disappeared after the decondensation was complete.

By TLC analyses of the human fertilization process, we confirmed the appearance of FC at the sperm entry point (SEP), 30 minutes after extrusion of the second polar body (PB) [2,3], and then evaluated the fertilization time course from insemination to disappearance of the FC (Figure 1). Considering that oocytes showing an FC were more likely to develop into a good quality embryo (GQE) than those without FC, the appearance of FC may be associated with embryo quality (Table 1). Such physiological effects should be studied further in human fertilization.

Cytoplasmic flare (Flare) (Supplementary Movie 3): After introduction of the sperm centrosome following sperm incorporation, the radial sperm aster was formed by the polymerization of microtubules adjacent to the decondensing male pronucleus ( $\mathrm{mPN})$. The female pronucleus ( $\mathrm{fPN}$ ) then migrated toward the $\mathrm{mPN}$ and both PN abutted [9]. Payne et al. [1] first showed by time-lapse video recording that the Flare radiated out from the center of the oocyte with ICSI. In our TLC, the Flare was clearly observed emanating from the site where the FC disappeared and migrating toward the ooplasm cortex. Then, the fPN moved toward the mPN until the two PN abutted (Figure 2). We then examined the relationship between appearance of the Flare and movement of the fPN toward the mPN in oocytes with IVF $(n=29)$ and ICSI $(n=66)$ (Figure 3$)$. In the 25 IVF oocytes showing normal fertilization $(2 \mathrm{PN} / 2 \mathrm{~PB})$ and the 4 fertilized abnormally $(>3 \mathrm{PN} / 2 \mathrm{~PB})$, the Flare appeared and the fPN moved toward the mPN. In the ICSI oocytes, 63 of the 66 showed the Flare and the remaining did not, and in those with the Flare, 59 showed normal fertilization (2PN/2PB) and 4 showed abnormal fertilization. Thus, all IVF and ICSI oocytes with the Flare showed migration of the $\mathrm{PPN}$ toward the $\mathrm{mPN}$, while three ICSI oocytes without the Flare showed neither both PNs nor normal fertilization. The Flare therefore seemed to represent a visual manifestation of the sperm aster.

Nucleolar precursor body (NPB) (Supplementary Movie 4): The nucleolar precursor bodies (NPBs) are condensed components responsible for ribosomal assembly [10] and the production of some

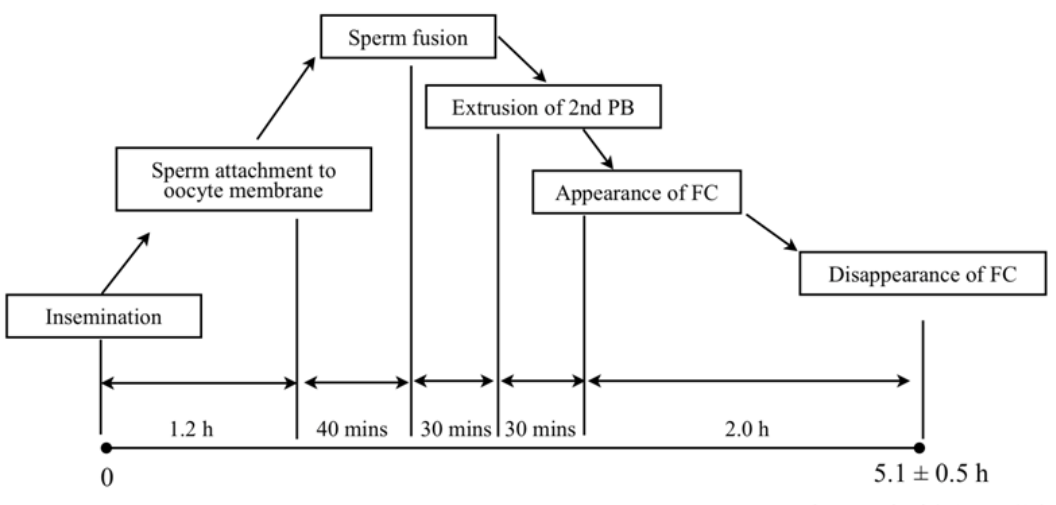

Figure 1: Time course from insemination to disappearance of FC.


Figure 2: Cytoplasmic flare and PN migration observed by TLC. 


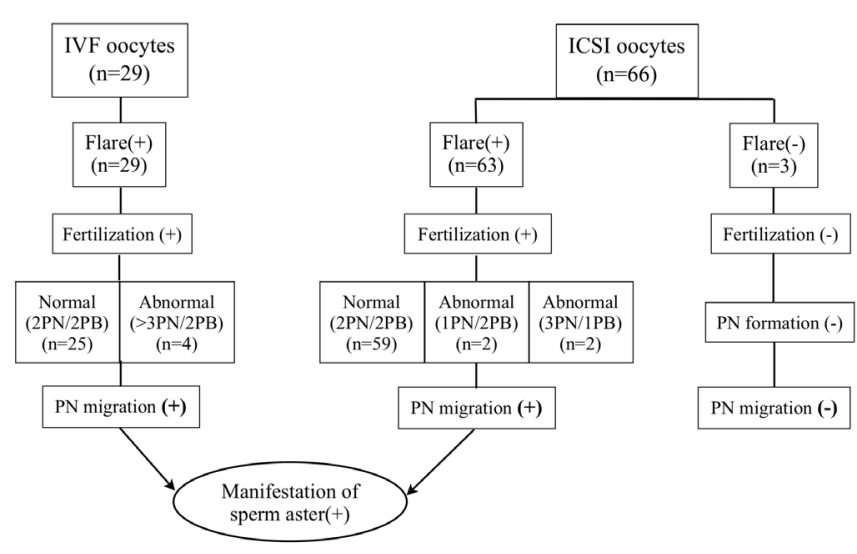

Figure 3: Relationship between cytoplasmic flare and PN migration in oocytes with IVF and ICSI.

\begin{tabular}{|l|c|c|c|c|}
\hline & \multicolumn{2}{|c|}{ GQE } & \multicolumn{2}{c|}{ PQE } \\
\hline $\begin{array}{l}\text { FC (+) } \\
(n=13)\end{array}$ & $76.9 \%$ & $(10 / 13)$ & $23.1 \%$ & $(3 / 13)$ \\
\hline $\begin{array}{l}\text { FC (-) } \\
(n=21)\end{array}$ & $42.9 \%$ & $(9 / 21)$ & $57.1 \%$ & $(12 / 21)$ \\
\hline
\end{tabular}

Table 1: Comparison of embryo quality in oocytes with/without FC. GQE: good quality embryos, PQE: poor quality embryos Out of 34 oocytes in which we could observe the entire fertilization process and the SEP, $13(38.2 \%)$ had a FC and the remainder showed no FC. Of 13 oocytes with FC, 10 (76.9\%) developed into a good quality embryo (GQE), while only 9 of 21 oocytes without FC $(42.9 \%)$ developed a GQE.

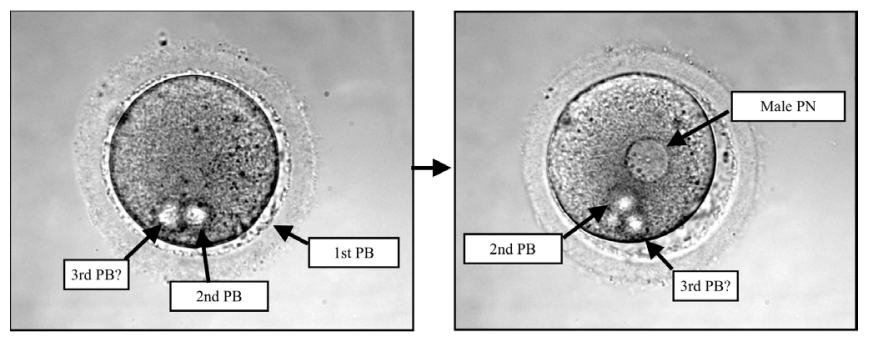

Figure 4: Novel mechanism of single-PN zygote formation after ICSI.

growth factors and developmental regulatory proteins [11]. NPBs are therefore likely to play important biological roles in early embryonic development. Several studies have correlated NPBs with embryo quality [12-14]. However, our TLC analysis revealed the distributions of NPBs to be quite dynamic with polarity changes from time to time, which made it extremly difficult to predict embryo quality based on NPBs in a single observation. Therefore, we evaluated the number of NPBs just after the PN appeared and before it disappeared. Just after the PN appeared, the average NPB number in GQEs and PQEs was 7.5 and 6.6 in male PN (mPN) and 6.1 and 4.9 in female PN (fPN), respectively, whereas immediately before the PN disappeared, NPBs in GQE and PQE were 7.1 and 6.8 in $\mathrm{mPN}$, and 5.6 and 4.9 in $\mathrm{PPN}$, respectively. Thus, we found no correlation between NPB number and embryo quality. However, the average number of NPB in mPN was higher than that in fPN [1]. These results suggest that evaluation of NPBs is not a useful biomarker of embryonic quality.

Translucent zone in peripheral ooplasm (cytoplasmic halo) (Supplementary Movie 5): The $\mathrm{mPN}$ and $\mathrm{fPN}$ was formed at 6.6 hours and 6.8 hours, respectively, after fertilization [3]. As they migrated toward the center of ooplasm and abutted (8.8 hours after the insemination), a peripheral cytoplasmic translucent zone called the cytoplasmic halo was formed. Prior to syngamy, the halo disappeared and the first cleavage division commenced. This cytoplasmic halo is thought to be the consequence of microtubule-mediated withdrawal of mitochondria and other cytoplasmic components to the perinuclear regions [15], and this hypothesis matches a sequence of cytoplasmic dynamics captured by TLC.

\section{Aberrant behavior of the fPN}

1PN zygote after ICSI (Supplementary Movie 6): Our TLC observation of ICSI oocytes revealed for the first time a novel phenomenon in which a third PB-like material was extruded in the perivitteline space shortly after extrusion of the second PB (Figure 4). These oocytes developed into single-PN zygotes and poor-quality, cleaved embryos. Until now, several mechanisms have been proposed for the formation of the 1PN zygote after IVF or ICSI, as follows: 1) parthenogenetic activation $[16-18,20], 2)$ asynchronous pronucleus formation [18-20], and 3) early fusion prior to syngamy [21]. However, our TLC study demonstrated a novel candidate mechanism in which ICSI oocytes extruded all chromosomal material including the $\mathrm{PPN}$, leaving only $\mathrm{mPN}$ injected. Indeed, this is quite an aberrant phenomenon, and there is no background information to support such an occurrence. However, we observed this eventuality in two separate movies by TLC.

Uneven 2PN zygote (Supplementary Movie 7): Our TLC analyses also revealed other aberrant phenomena in the formation of uneven 2PN zygotes after ICSI. As seen in the 1PN zygote formed after ICSI, a third PB-like matter was extruded in addition to the first and second $\mathrm{PB}$, and it appeared to contain a small PN-like body, formed after the $\mathrm{mPN}$ formed in the ooplasm. The PN-like body was then absorbed into the ooplasm and migrated toward the $\mathrm{mPN}$, leading to formation of an uneven 2PN embryo (Figure 5). Although it has been reported that zygotes with uneven 2PN may correlate with ooplasmic immaturity and chromosomal abnormalities [22,23], the etiology and physiological significance of those zygotes are still unknown. The TLC analyses in this study demonstrated for the first time a possible mechanism for the formation of uneven $2 \mathrm{PN}$ zygotes.

\section{Fragmentation (Supplementary Movie 8)}

It is well known that embryo fragmentation or the generation of anucleate cell fragments is an important biomarker of embryo quality during early embryonic development after ART [24,25]. Several hypotheses regarding embryo fragmentation have been reported such as programmed cell death (apoptosis) [26], telomere length [27], increased maternal age [28], abnormalities in cytoskeleton and microtubule organization $[29,30]$. However, the precise underlying mechanism remains controversial and we considered our TLC observation to be a powerful tool to elucidate the dynamic morphology of this phenomenon.

We obtained one of the best TLC movies showing embryo fragmentation after ICSI. After the cytoplasmic flare appeared from the center of the ooplasm toward the cortex, $\mathrm{mPN}$ was formed in the center of the ooplasm, followed by the appearance of fPN just beneath the first and second PB. The fPN migrated quickly toward the mPN and abutted. In 17 hours and 20 minutes after the commencement of TLC observation, this zygote reached syngamy. Two hours later, first cleavage started and was completed within 30 minutes. During the division, a few fragments appeared along the cleavage furrow and 


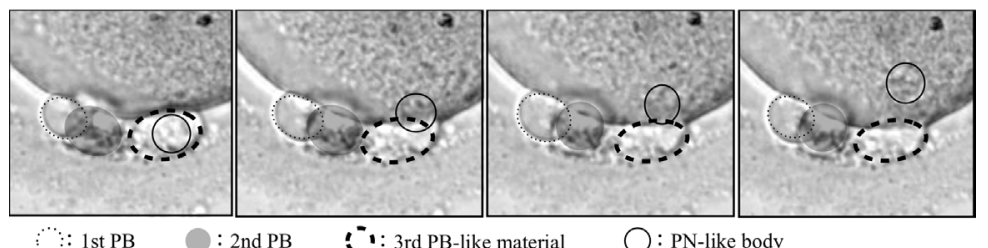

Figure 5: Novel mechanism of uneven 2PN zygote after ICSI. After the second PB was extruded, a third PB-like material was also extruded. At the same time as $\mathrm{mPN}$ formation in the ooplasm, a small fPN-like body was also formed within the third PB-like material and then resorbed into the ooplasm to migrate toward the mPN.
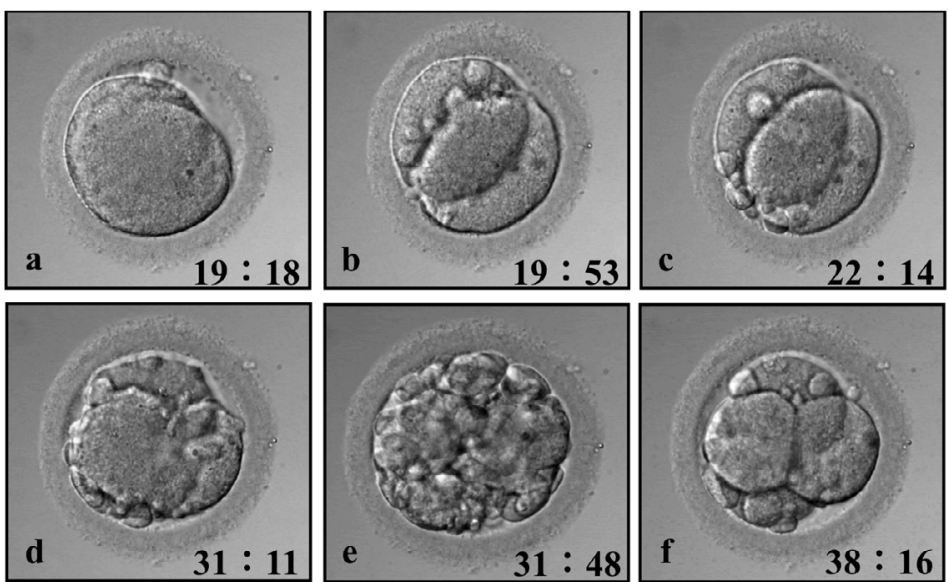

Figure 6: Fragmentation and $1^{\text {st }}$ and $2^{\text {nd }}$ cleavage. After syngamy, the first cleavage furrow appeared (a) During first cleavage, some fragments were generated along the furrow (b) but the number of fragments decreased about 2.5 hours later (c) About 10 hours later, the second cleavage furrow appeared (d) and the blastomere shape degraded into poor quality during the middle of the second cleavage (e) However, about 6 hours later, the embryo developed into good quality.

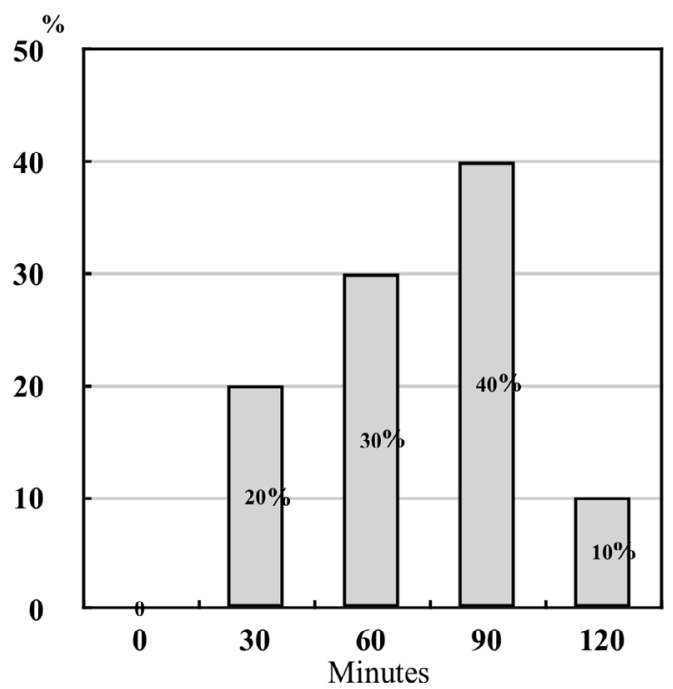

Figure 7: Time after $1^{\text {st }} / 2^{\text {nd }}$ cleavage and resorption of fragments.

then decreased in number as the time passed. At 31 hours of TLC observation, the second cleavage commenced and the embryonic morphology dramatically changed into poor quality by 31 hours and 48 minutes. However, at 33 hours, the blastomere shape changed into good quality. More fragments were observed during the second cleavage process than during the first cleavage (Figure 6).
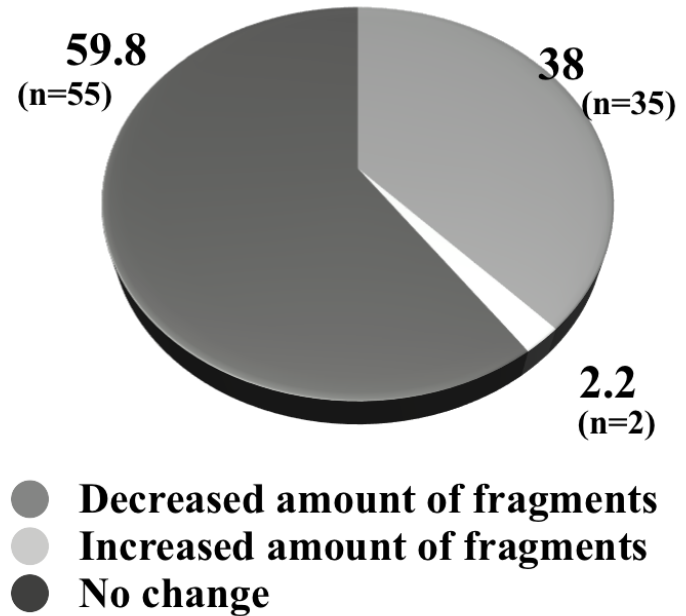

Figure 8: Qualitative change in the amount of fragments after the $1^{\text {st }} / 2^{\text {nd }}$ cleavage.

In addition, we also confirmed that fragmentation occurred during cytokinesis and was not observed at any other time during the cell cycle. Some of the fragments were resorbed into the blastomere in the first 2 hours following both first and second cleavage (Figures 7 and 8).

\section{Blastocoel collapse (Supplementary Movie 9)}

Using donated frozen/thawed 4-cell embryos (IVF; $\mathrm{n}=21$, ICSI; 


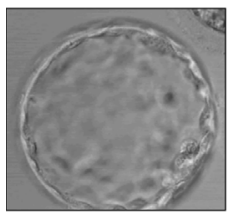

88 h 19 mins

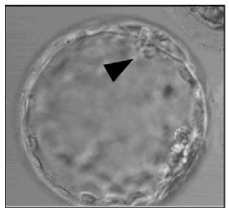

91 h 59 mins

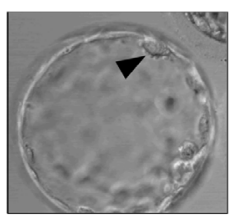

90 h 44 mins

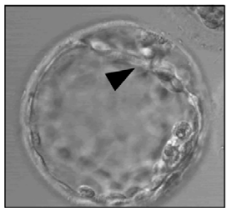

$92 \mathrm{~h} 04 \mathrm{mins}$

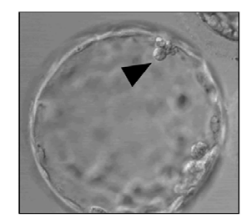

90 h 59 mins



$94 \mathrm{~h} 34 \mathrm{mins}$

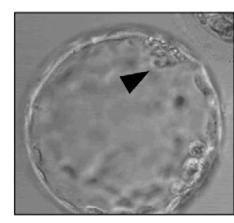

91 h 34 mins

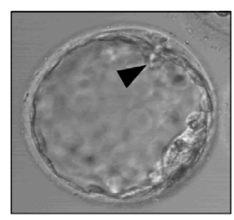

94 h 39 mins

Figure 9: Disturbance of trophectoderm and blastocoel collapse (arrow).

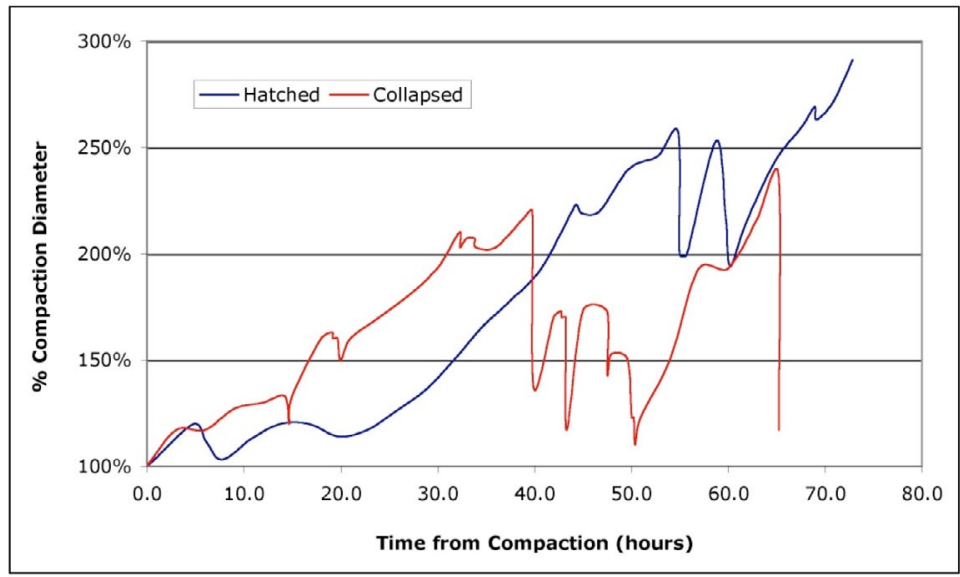

Figure 10: Time course of the diameter of blastocoel cavities in two embryos.

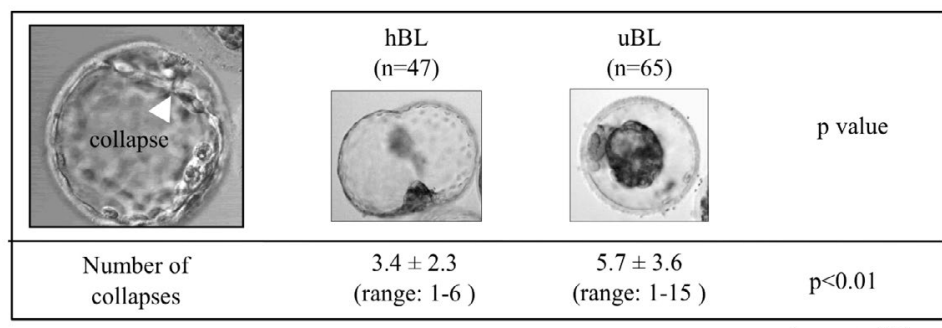

hBL: hatched blastocyst, uBL: unhatched blastocyst

$($ mean \pm SD)

Figure 11: Rate of blastocoel collapse and embryonic outcome.

$\mathrm{n}=13$ ), TLC observation was carried out for up to 5 days. One of these TLC images showed a novel mechanism for blastocoel collapse. During the expanding blastocyst stage, a localized part of trophectoderm (TE) became suddenly disturbed followed by blastocoel collapse. After that event, while the TE was repaired, the blasotocoel cavity was quickly re-expanded (Figure 9).

Furthermore, we compared the time course of two typical embryos; one was a hatched embryo and the other was an unhatched embryo showing degeneration (Figure 10). The hatched embryo developed slowly and steadily without the collapse of TE until hatching was complete. Contrarily, the unhatched embryo developed quickly, showed a number of blastocoel collapses, and finally degenerated. We compared the relationship between the number of collapses and culture outcome in two types of blastocysts [hatched (hBL) vs. unhatched $(\mathrm{uBL})]$ (Figure 11), and found a significantly higher number in uBL than in $\mathrm{hBL}(\mathrm{P}<0.01)$. Therefore, although the nature of normality and/or differences in blastocoel collapse between in vivo and in vitro conditions remain controversial $[25,26]$, we concluded that the blastocoel collapse is a super-physiological phenomenon and may be indeed be an artifact of extended culture in vitro. 




Figure 12: Inward pattern

After the blastocyst reached the fully expanded stage (a), the blastocoel cavity suddenly collapsed and a break in the ZP at 1 o'clock (b,c). The blastocyst then escaped from the site of the break in the ZP (d-h).
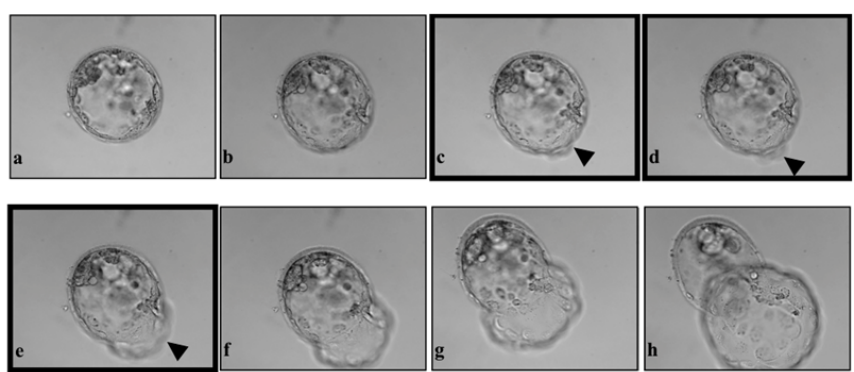

Figure 13: Outward pattern

The blastocyst fully expanded (a,b) and the ZP at 5 o'clock ruptured without collapse (c-e). The expanded blastocyst then quickly escaped from the ZP (f-h).

\begin{tabular}{|l|l|l|l|l|}
\hline & $\begin{array}{l}\text { collapse(-) } \\
\text { (n=9; 27.3\%) }\end{array}$ & $\begin{array}{l}\text { minor } \\
\text { collapse } \\
\text { (n=17; 51.5\%) }\end{array}$ & $\begin{array}{l}\text { major } \\
\text { collapse } \\
(\mathbf{n = 7 ; 2 1 . 2 \% )}\end{array}$ & p \\
\hline $\begin{array}{l}\text { Time required } \\
\text { for hatching } \\
\text { (hrs) }\end{array}$ & $6.1 \pm 2.8^{* a}$ & $7.5 \pm 2.8$ & $9.9 \pm 3.1^{* b}$ & $p<0.05$ \\
\hline
\end{tabular}

Major collapse indicated $\geqq 30 \%$ of blastocoel cavity

Table 2: Degree of blastocoel collapse and time required for hatching.

\section{Hatching process (Supplementary Movie 10 and 11)}

Escape from the ZP of the expanded blastocyst (hatching) is an epilogue in embryonic development before implantation. Although several hypotheses have been proposed regarding the mechanism of hatching in mouse, bovine, equine, and human [33-35], only limited information exists about the entire process. Using donated day-2 frozen/thawed human embryos, we looked at embryonic development up to hatching by TLC $[2,3]$. In this analyses, we found another novel phenomenon regarding the hatching pattern of human expanded blastocysts in that two patterns exist, which we named the "inward" and "outward" patterns. In the "inward" pattern, after the blastocyst reached the fully expanded stage, the blastocoel cavity suddenly collapsed and, at the same time, the ZP retracted inwardly followed by a break in the ZP, through which the blastocyst then escaped (Figure 12).

In the "outward" pattern, after the blastocoel cavity expanded steadily without any blastocoel collapse, the ZP was broken and the blastocyst escaped quickly from the break site (Figure 13). Of the expanded blastocysts that escaped successfully from the ZP $(n=33)$, only $9(27.3 \%)$ showed no blastocoel collapse when the blastocysts went through the $\mathrm{ZP}$, and this occurrance was irrespective of the grade and frequency of blastocoel collapse. In addition, the time required for escape from the ZP in blastocysts without blastocoel collapse was significantly less than that in blastocysts showing collapse $(\mathrm{P}<0.05)$ (Table 2). Blastocysts with fewer blastocoel collapses could escape from the ZP quicker than those with an increased number of collapses.
Although most of the expanded blastocysts escaped from the ZP showed blastocoel collapse under our in vitro culture conditions, this result suggested that blastocysts in vivo are more likely to follow the "outward" pattern, because in vitro culture conditions are still more consistent than the in vivo situation.

In conclusion, herein we presented novel in vitro data and information regarding human embryonic development observed by using TLC. This microscopy system enables precise visual analyses of human embryonic behavior, and the knowledge derived from using the system provides novel and important information about both the physiology of embryonic development and the quality of in vitro culture conditions to establish safer and more effective ART programs.

\section{Acknowledgements}

We would like to express particular thanks to Dianna Payne and Sean P Flaherty in Adelaide, Australia for their invaluable support in developing a new in vitro culture system for time-lapse cinematography and in research collaborations. We would like to especially thank Mr Nobukazu Murakami for image editing. This work was also supported by all staff in the Reproductive Centre, Mio Fertility Clinic.

\section{References}

1. Payne D, Flaherty SP, Barry MF, Matthews CD (1997) Preliminary observations on polar body extrusion and pronuclear formation in human oocytes using timelapse video cinematography. Hum Reprod 12: 532-541.

2. Mio $Y$ (2006) Morphological analysis of human embryonic development using time-lapse cinematography. J Mamm Ova Res 23: 27-36.

3. Mio Y, Maeda K (2008) Time-lapse cinematography of dynamic changes occurring during in vitro development of human embryos. Am J Obstet Gynecol 199: 660.e1-5.

4. Mio Y, Iwata K, Yumoto K, Kai Y, Sargant HC, et al. (2012) Possible mechanism of polyspermy block in human oocytes observed by time-lapse cinematography. J Assist Reprod Genet 29: 951-956.

5. Mcculloh DH, Lynn JW, Chambers EL (1987) Membrane depolarization facilitates sperm entry, large fertilization cone formation, and prolonged current responses in sea urchin oocytes. Dev Biol 124: 177-190. 
Citation: Mio Y, Iwata K, Yumoto K, Maeda K (2014) Human Embryonic Behavior Observed with Time-Lapse Cinematography. J Health Med Informat 5: 143. doi:10.4172/2157-7420.1000143

6. Lynn JW, McCulloh DH, Chambers EL (1988) Voltage clamp studies of fertilization in sea urchin eggs. Current patterns in relation to sperm entry, nonentry, and activation. Dev Biol 128: 305-323.

7. Johnson $\mathrm{MH}$, Pickering SJ, Flach G (1984) Changes in actin distribution during fertilization of the mouse egg. J Embryol Exp Morphol 237: 211-237.

8. Piotrowska K, Zernicka-Goetz M (2001) Role for sperm in spatial patterning of the early mouse embryo. Nature 409: 517-521.

9. Simerly C, Wu GJ, Zoran S, Ord T, Rawlins R, et al. (1995) The paternal inheritance of centrosome, the cell's microtubule organizing center, in humans, and the implications for infertility. Nat Med 1: 47-52.

10. Tesarik, J, Kopency, V (1990) Assembly of the nucleolar precursor bodies in human male pronuclei is correlated with an early RNA synthetic activity. Exp Cell Res 191: 153-156.

11. Pederson T (1998) Growth factors in the nucleolus? J Cell Biol 143: 279-281.

12. Cummins JM, Breen TM, Harrison KL, Shaw JM, Wilson LM, et al. (1986) A formula for scoring human embryo growth rates in in vitro fertilization: its value in predicting pregnancy and in comparison with visual estimates of embryo quality. In Vitro Fert Embryo Transf 3: 284-295.

13. Tesarik J, Greco E (1999) The probability of abnormal preimplantation development can be predicted by a single static observation on pronuclear stage morphology. Human Reprod 14: 1318-1323.

14. Scott L, Alvero R, Leondires M, Miller B (2000) The morphology of human pronuclear embryos is positively related to blastocyst development and implantation. Hum Reprod 15: 2394-2403.

15. Van Blerkom J, Davis P, Alexander S (2000) Differential mitochondria distribution in human pronuclear embryos leads to disproportionate inheritance between blastomeres: relationship to microtubular organization, ATP content and competence. Hum Reprod 15: 2621- 2633

16. Balakier H, Squire J, Casper RF (1993) Characterization of abnorma one pronuclear human oocytes by morphology, cytogenetics and in-situ hybridization. Hum Reprod 8: 402-408.

17. Taylor AS, Braude PR (1994) The early development and DNA content of activated human oocytes and parthenogenetic human embryos. Hum Reprod 9: 2389-2397.

18. Sultan KM, Munne S, Palermo GD, Alikani M, Cohen J (1995) Chromosoma status of uni-pronuclear human zygotes following in-vitro fertilization and intracytoplasmic sperm injection. Hum Reprod 10: 132-136

19. Nagy ZP, Liu J, Joris H, Devroey P, Van Steirteghem A (1994) Time-course of oocyte activation, pronucleus formation and cleavage in human oocytes fertilized by intracytoplasmic sperm injection. Hum Reprod 9: 1743-1748.

20. Staessen C, Janssenswillen C, Devroey P, Van Steirteghem AC (1993) Cytogenetic and morphological observations of single pronucleated human oocytes after in-vitro fertilization. Hum Reprod 8: 221-223.

21. Leveron J, Munne S, Willadsen S, Rosenwaks Z,and Cohen J (1995) Male and female genomes associated in a single pronucleus in human zygotes. Biol Reprod 52: 653-657

22. Sadowy S, Tomkin G, Munne S, Ferrara-Congedo T, Cohen J (1998) Impaired development of zygotes with uneven pronuclear size. Zygote 63: 137-141.

23. Manor D, Drugan A, Stein D, Pillar M,Itskovitz-Eldor J (1999) Unequal pronuclear size-A powerful predictor of embryonic chromosome anomalies. J Assist Reprod Genet 16: 385-389.

24. Fujimoto VY, Browne RW, Bloom MS, Sakkas D, Alikani M (2010) Pathogenesis, developmental consequences, and clinical correlations of human embryo fragmentation. Fertil Steril 95: 1197-1204.
25. Alpha scientists in reproductive medicine and ESHRE special interest group embryology (2011) Istanbul consensus workshop on embryo assessment proceedings of an expert meeting. Hum Reprod 26: 1270-1283.

26. Jurisicova A, Varmuza S, Casper RF (1996) Programmed cell death and human embryo fragmentation. Mol Hum Reprod 2: 93-98.

27. Keefe DL, Franco S, Liu L, Trimarchi J, Cao B, et al. (2005) Telomere length predicts embryo fragmentation after in vitro fertilization in women--toward a telomere theory of reproductive aging in women. Am J Obstet Gynecol 192 $1256-1260$.

28. Ziebe S, Loft A, Petersen JH, Andersen AG, Lindenberg S, et al. (2001) Embryo quality and developmental potential is compromised by age. Acta Obstet Gynecol Scand 80: 169-174.

29. Alikani M (2005) Epithelial cadherin distribution in abnormal human preimplantation embryos. Hum Reprod 20: 3369-3375.

30. Liu L, Keefe DL (2002) Ageing-associated aberration in meiosis of oocytes from senescence-accelerated mice. Hum Reprod 17: 2678-2685.

31. Niimura S (2003) Time-lapse videomicrographic analyses of contractions in mouse blastocysts. J Reprod Dev 49: 413-423.

32. Checiu I, Checiu M (1996) There are no in vivo pulsations of mouse blastocysts. Rom J Morphol Embryol 42: 147-154.

33. Yamazaki K, Suzuki R, Hojo E, Kondo S, Kato Y, et al. (1994) Trypsin-like hatching enzyme of mouse blastocysts: Evidence for its participation in hatching process before zona shedding of embryos. Develop Growth and Differ 36: $149-154$

34. Thomas M, Jain S, Kumar GP, Laloraya M (1997) A programmed oxyradical burst causes hatching of mouse blastocysts. J Cell Sci 110: 1597-1602.

35. Gonzales DS, Jones JM, Pinyopummintr T, Carnevale EM, Ginther OJ, et al. (1996) Trophectoderm projections: a potential means for locomotion, attachemnt and implantation of bovine, equine and human blastocysts. Hum Reprod 11: 2739-2745. 